Supporting Information for

\title{
Water Stable Heterometallic Zn-Tb Coordination Polymer for Rapid Detection of the Ultraviolet Filter Benzophenone
}

Qiutong Xu, Zhonghang Chen, Hui Min, Fen Song, Yu-Xia Wang, Wei Shi, * and Peng Cheng*

Key Laboratory of Advanced Energy Materials Chemistry (MOE), College of Chemistry, Nankai University, Tianjin 300071, China.

*To whom correspondence should be addressed. E-mail: shiwei@nankai.edu.cn;

pcheng@nankai.edu.cn

\section{Contents}

Table S1 Crystal data and structural refinement parameters for $\mathbf{1}$ and $\mathbf{2 .}$

Table S2 Selected bond lengths $(\AA)$ and angles $\left(^{\circ}\right)$ for $\mathbf{1}$ and $\mathbf{2}$.

Figure S1 Fourier transform infrared spectra of $\mathbf{1}$ and $\mathbf{2}$.

Figure S2 Experimental and simulated PXRD patterns of $\mathbf{1}$ and $\mathbf{2}$.

Figure S3 PXRD patterns of 2 before and after storage in water for $48 \mathrm{~h}$.

Figure S4 PXRD patterns of 2 at $\mathrm{pH}$ values from 4 to 9 in $\mathrm{HCl} / \mathrm{NaOH}$ solutions.

Figure S5 Thermogravimetric analysis curves of $\mathbf{1}$ and $\mathbf{2}$.

Figure S6 Decay curves and luminescence lifetime of 1 monitored at $546 \mathrm{~nm}$.

Figure S7 Decay curves and luminescence lifetimes of 2 monitored at $546 \mathrm{~nm}$ with different concentrations of BP.

Figure S8 UV-vis absorption spectra of the $\mathrm{H}_{2} \mathrm{~L}$ ligand and BP.

Figure S9 Phosphorescence spectrum of $\left\{\left[\mathrm{Gd}_{2} \mathrm{Zn}(\mathrm{L})_{4}\left(\mathrm{H}_{2} \mathrm{O}\right)_{8}\right] \cdot 8 \mathrm{H}_{2} \mathrm{O}\right\}_{n}$ at $77 \mathrm{~K}$.

Figure S10 CIE 1931 chromaticity diagrams of 1 and 2.

Figure S11 Emission spectra of $\mathbf{2}$ before and after storage in water for $48 \mathrm{~h}$.

Figure S12 Emission intensities of 2 recorded at $\mathrm{pH} 4-9$ with immersion in 
$\mathrm{HCl} / \mathrm{NaOH}$ solution and various time intervals containing BP.

Figure S13 PXRD patterns of $\mathbf{2}$ before and after detection of BP.

Figure S14 Reproducibility of the quenching ability of $\mathbf{2}$ dispersed in water in the presence of BP with six continuous quenching cycles at $546 \mathrm{~nm}$, and the ratio of the luminescence intensity of 2 at $546 \mathrm{~nm}$ before and after addition of BP. 
Table S1 Crystal data and structural refinement parameters for $\mathbf{1}$ and $\mathbf{2}$.

\begin{tabular}{|c|c|c|}
\hline & 1 & 2 \\
\hline Formula & $\mathrm{C}_{38} \mathrm{H}_{53} \mathrm{Cl}_{3} \mathrm{TbN}_{7} \mathrm{O}_{13}$ & $\mathrm{C}_{28} \mathrm{H}_{40} \mathrm{Cl}_{4} \mathrm{~Tb}_{2} \mathrm{ZnN}_{4} \mathrm{O}_{32}$ \\
\hline$F_{\mathrm{W}}$ & 1081.14 & 1469.65 \\
\hline Crystal system & Trigonal & Monoclinic \\
\hline Space group & $P 3_{2}$ & $C 2 / c$ \\
\hline$a / \AA$ & $13.5444(10)$ & $25.1044(5)$ \\
\hline$b / \AA$ & $13.5444(10)$ & $15.5880(3)$ \\
\hline$c / \AA$ & $22.4338(4)$ & $12.3986(3)$ \\
\hline$\alpha\left(^{\circ}\right)$ & 90 & 90.00 \\
\hline$\beta\left(^{\circ}\right)$ & 90 & $96.797(2)$ \\
\hline$\gamma\left({ }^{\circ}\right)$ & 120 & 90.00 \\
\hline$V / \AA^{3}$ & $3564.12(8)$ & $4817.81(18)$ \\
\hline$Z$ & 3 & 4 \\
\hline$\rho\left(\mathrm{g} \mathrm{cm}^{-3}\right)$ & 1.511 & 2.026 \\
\hline$\mu\left(\mathrm{mm}^{-1}\right)$ & 9.430 & 3.723 \\
\hline$F(000)$ & 1650.0 & 2880.0 \\
\hline$R_{\text {int }}$ & 0.0428 & 0.0538 \\
\hline GOF on $F^{2}$ & 1.049 & 1.015 \\
\hline$R_{1}^{\mathrm{a}}, w R_{2}^{\mathrm{b}}(I \geq 2 \sigma(I))$ & $0.0582,0.1571$ & $0.0411,0.0696$ \\
\hline$R_{1}{ }^{\mathrm{a}}, w R_{2}^{\mathrm{b}}$ (all data) & $0.0589,0.1589$ & $0.0620,0.0770$ \\
\hline
\end{tabular}


Table S2 Selected bond lengths $(\AA)$ and angles $\left(^{\circ}\right)$ for $\mathbf{1}$ and $\mathbf{2}$.

\begin{tabular}{|c|c|c|c|c|c|}
\hline \multicolumn{6}{|c|}{1} \\
\hline Tb1-O5 & $2.369(9)$ & O5-Tb1-O9 & $78.4(4)$ & $\mathrm{O} 7-\mathrm{Tb} 1-\mathrm{O} 11$ & $79.2(4)$ \\
\hline Tb1-O9 & $2.410(10)$ & $\mathrm{O} 5-\mathrm{Tb} 1-\mathrm{O} 3$ & $144.5(4)$ & $\mathrm{O} 3-\mathrm{Tb} 1-\mathrm{N} 3$ & $70.7(4)$ \\
\hline Tb1-O7 & $2.366(9)$ & O5-Tb1-N3 & $73.9(3)$ & O3-Tb1-N1 & $63.2(3)$ \\
\hline $\mathrm{Tb} 1-\mathrm{O} 3$ & $2.435(8)$ & $\mathrm{O} 5-\mathrm{Tb} 1-\mathrm{N} 2$ & $64.83(3)$ & $\mathrm{O} 1-\mathrm{Tb} 1-\mathrm{O} 11$ & $143.7(4)$ \\
\hline $\mathrm{Tb} 1-\mathrm{O} 11$ & $2.396(10)$ & O5-Tb1-N1 & $135.5(3)$ & O11-Tb1-O9 & $128.2(3)$ \\
\hline $\mathrm{Tb} 1-\mathrm{N} 3$ & $2.470(6)$ & O9-Tb1-N3 & $64.0(3)$ & O1-Tb1-O9 & $81.4(4)$ \\
\hline Tb1-O1 & $2.376(10)$ & O9-Tb1-N2 & $137.3(3)$ & $\mathrm{O} 1-\mathrm{Tb} 1-\mathrm{N} 2$ & $72.4(3)$ \\
\hline Tb1-N3 & $2.470(6)$ & O9-Tb1-N1 & $69.7(3)$ & $\mathrm{O} 1-\mathrm{Tb} 1-\mathrm{O} 3$ & $128.0(4)$ \\
\hline \multirow[t]{6}{*}{$\mathrm{Tb} 1-\mathrm{N} 1$} & $2.491(7)$ & $\mathrm{O} 9-\mathrm{Tb} 1-\mathrm{O} 3$ & $85.3(3)$ & $\mathrm{O} 1-\mathrm{Tb} 1-\mathrm{N} 3$ & $140.1(3)$ \\
\hline & & $\mathrm{O} 7-\mathrm{Tb} 1-\mathrm{N} 2$ & $63.8(3)$ & $\mathrm{O} 1-\mathrm{Tb} 1-\mathrm{N} 1$ & $65.0(3)$ \\
\hline & & $\mathrm{O} 7-\mathrm{Tb} 1-\mathrm{O} 5$ & $128.6(3)$ & & \\
\hline & & O7-Tb1-O9 & $146.3(4)$ & & \\
\hline & & $\mathrm{O} 7-\mathrm{Tb} 1-\mathrm{O} 3$ & $80.3(4)$ & & \\
\hline & & $\mathrm{O} 7-\mathrm{Tb} 1-\mathrm{O} 1$ & $83.8(4)$ & & \\
\hline
\end{tabular}

\begin{tabular}{|c|c|c|c|c|c|}
\hline \multicolumn{6}{|c|}{2} \\
\hline Tb1-O4 & $2.470(4)$ & $\mathrm{O} 4-\mathrm{Tb} 1-\mathrm{N} 1$ & $63.80(14)$ & $\mathrm{O} 10-\mathrm{Tb} 1-\mathrm{N} 2$ & $131.03(14)$ \\
\hline $\mathrm{Tb} 1-\mathrm{O} 4^{1}$ & $2.481(4)$ & $\mathrm{O} 4-\mathrm{Tb} 1-\mathrm{N} 2$ & $134.88(13)$ & $\mathrm{O} 7-\mathrm{Tb} 1-\mathrm{O} 4$ & $149.71(14)$ \\
\hline Tb1-O5 & $2.429(4)$ & $\mathrm{O} 5-\mathrm{Tb} 1-\mathrm{O} 4$ & $76.81(13)$ & $\mathrm{O} 7-\mathrm{Tb} 1-\mathrm{O} 4^{1}$ & $101.90(12)$ \\
\hline Tb1-O9 & $2.439(4)$ & $\mathrm{O} 5-\mathrm{Tb} 1-\mathrm{O} 4^{1}$ & $75.09(13)$ & O7-Tb1-N1 & $135.43(13)$ \\
\hline $\mathrm{Tb} 1-\mathrm{O} 10$ & $2.409(4)$ & $\mathrm{O} 5-\mathrm{Tb} 1-\mathrm{O} 7$ & $127.78(13)$ & $\mathrm{O} 7-\mathrm{Tb} 1-\mathrm{N} 2$ & $64.38(14)$ \\
\hline Tb1-O7 & $2.449(3)$ & $\mathrm{O} 5-\mathrm{Tb} 1-\mathrm{N} 1$ & $71.05(14)$ & $\mathrm{O} 1-\mathrm{Tb} 1-\mathrm{O} 4$ & $127.97(12)$ \\
\hline $\mathrm{Tb} 1-\mathrm{O} 1$ & $2.427(4)$ & $\mathrm{O} 5-\mathrm{Tb} 1-\mathrm{N} 2$ & $63.43(13)$ & $\mathrm{O} 1-\mathrm{Tb} 1-\mathrm{O} 5$ & $82.94(13)$ \\
\hline Tb1-N1 & $2.515(4)$ & $\mathrm{O} 9-\mathrm{Tb} 1-\mathrm{O} 4$ & $78.75(12)$ & O1-Tb1-O9 & $134.44(13)$ \\
\hline $\mathrm{Tb} 1-\mathrm{N} 2$ & $2.498(5)$ & O9-Tb1-N1 & $121.23(14)$ & $\mathrm{O} 1-\mathrm{Tb} 1-\mathrm{O} 7$ & $77.47(12)$ \\
\hline $\mathrm{Zn} 1-\mathrm{O} 12^{2}$ & $2.085(4)$ & O9-Tb1-N2 & $121.30(11)$ & O5-Tb1-O9 & $142.61(13)$ \\
\hline $\mathrm{Zn} 1-\mathrm{O} 12$ & $2.085(4)$ & $\mathrm{O} 10-\mathrm{Tb} 1-\mathrm{O} 4$ & $93.19(12)$ & $\mathrm{O} 1-\mathrm{Tb} 1-\mathrm{N} 1$ & $64.36(13)$ \\
\hline $\mathrm{Zn} 1-\mathrm{O}^{2}$ & $2.086(4)$ & $\mathrm{O} 10-\mathrm{Tb} 1-\mathrm{O} 5$ & $140.60(12)$ & $\mathrm{O} 1-\mathrm{Tb} 1-\mathrm{N} 2$ & $69.15(14)$ \\
\hline $\mathrm{Zn} 1-\mathrm{O} 8$ & $2.086(4)$ & O10-Tb1-O9 & $68.29(13)$ & $\mathrm{N} 2-\mathrm{Tb} 1-\mathrm{N} 1$ & $117.23(16)$ \\
\hline $\mathrm{Zn} 1-\mathrm{O} 11^{2}$ & $2.110(4)$ & $\mathrm{O} 10-\mathrm{Tb} 1-\mathrm{O} 7$ & $77.64(12)$ & $\mathrm{O}^{2}-\mathrm{Zn} 1-\mathrm{O} 8$ & $88.1(2)$ \\
\hline $\mathrm{Zn} 1-\mathrm{O} 11$ & $2.110(4)$ & $\mathrm{O} 10-\mathrm{Tb} 1-\mathrm{N} 1$ & $70.38(13)$ & $\mathrm{O} 8-\mathrm{Zn} 1-\mathrm{O} 11$ & $175.51(15)$ \\
\hline
\end{tabular}

${ }_{1}^{1} 1 / 2-X, 1 / 2-Y,-Z ;{ }^{2} 1-X,+Y, 1 / 2-Z$. 


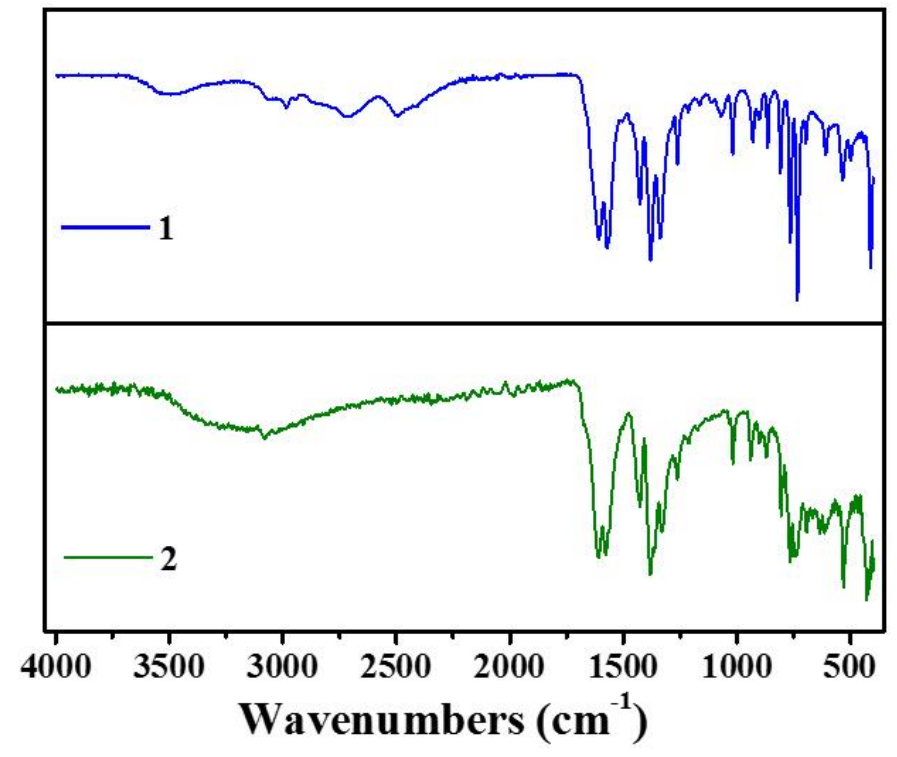

Figure S1 Fourier transform infrared spectra of $\mathbf{1}$ and 2. 
(a)

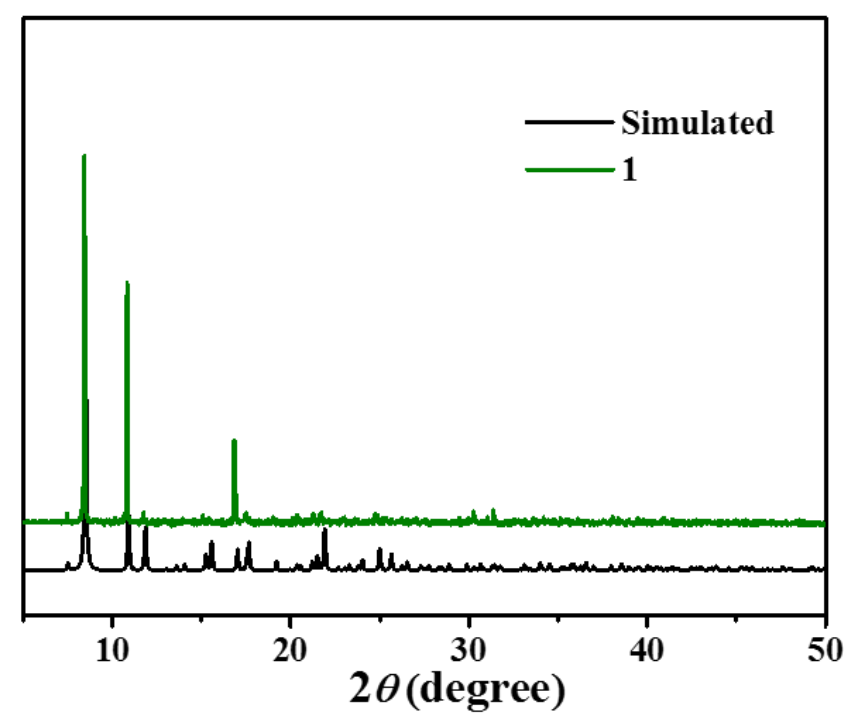

(b)

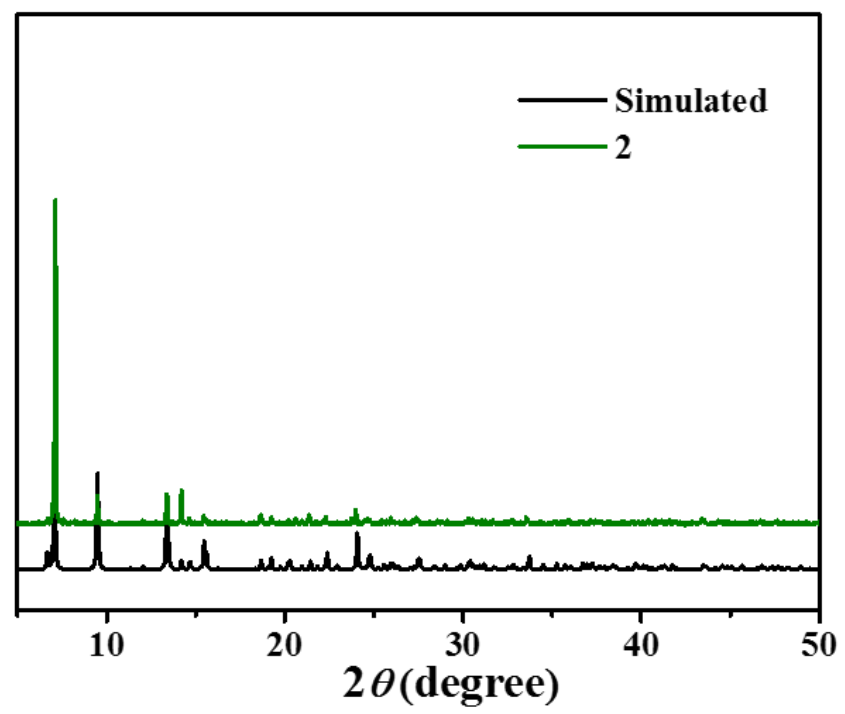

Figure S2 Experimental and simulated PXRD patterns of 1 (a) and 2 (b). 


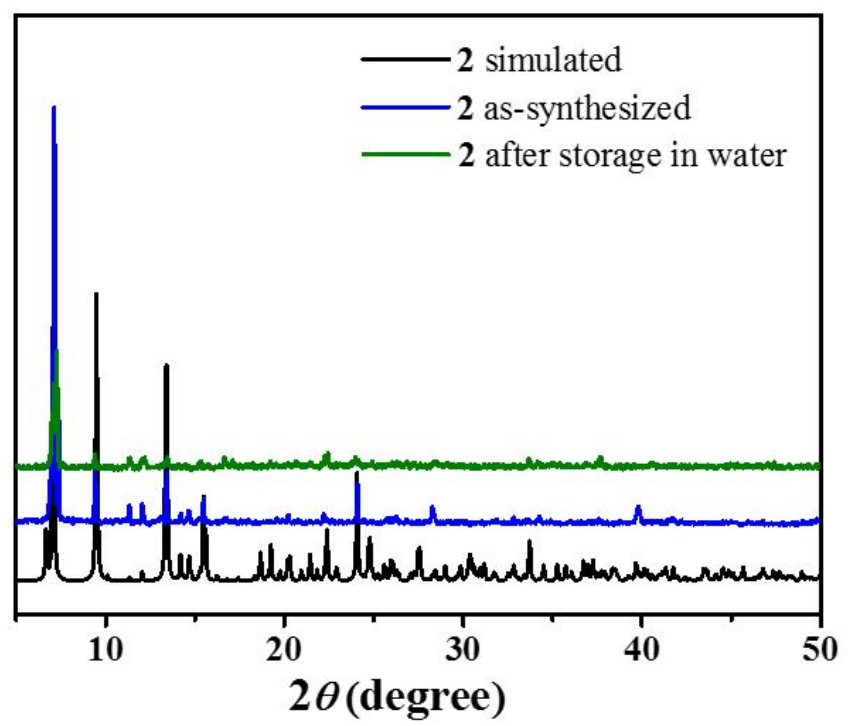

Figure S3 PXRD patterns of 2 before and after storage in water for $48 \mathrm{~h}$.

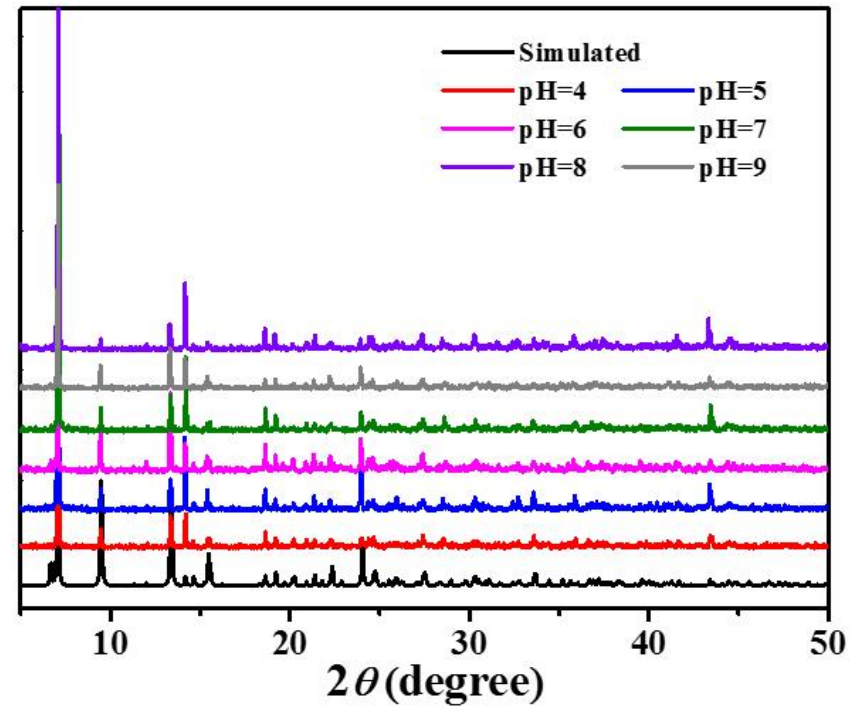

Figure S4 PXRD patterns of 2 at $\mathrm{pH}$ values from 4 to 9 in $\mathrm{HCl} / \mathrm{NaOH}$ solutions. 

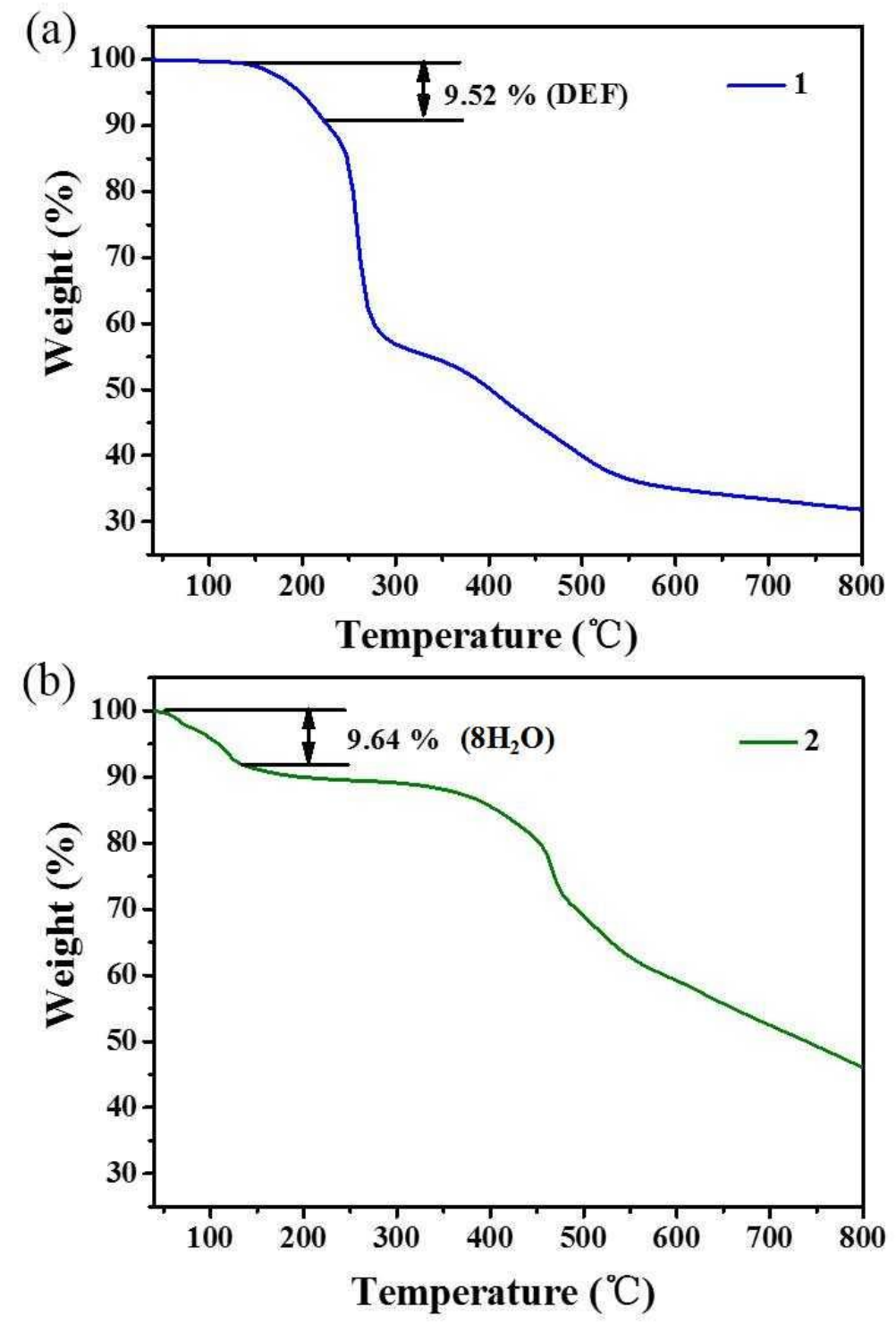

Figure S5 Thermogravimetric analysis curves of 1 (a) and 2 (b). 


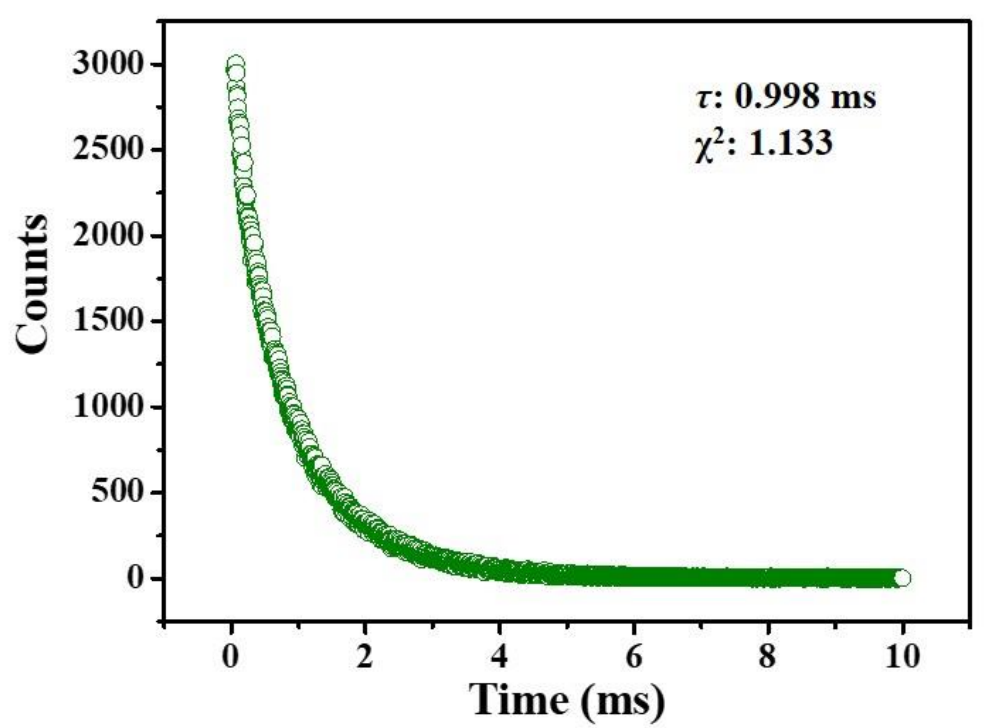

Figure S6 Decay curves and luminescence lifetime of 1 monitored at $546 \mathrm{~nm}$. 

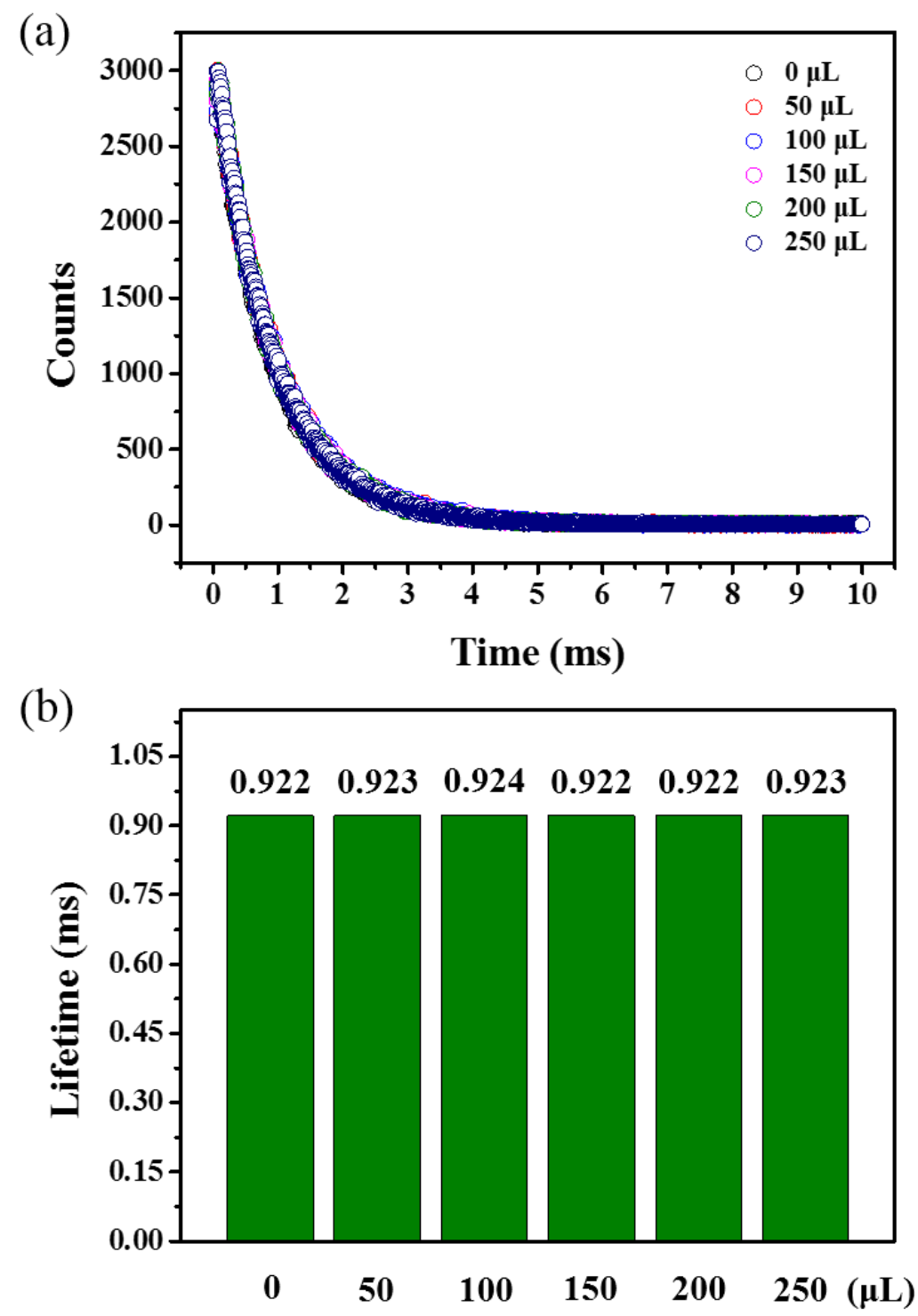

Figure S7 Decay curves (a) and luminescence lifetimes (b) of 2 monitored at $546 \mathrm{~nm}$ with different concentrations of BP. 


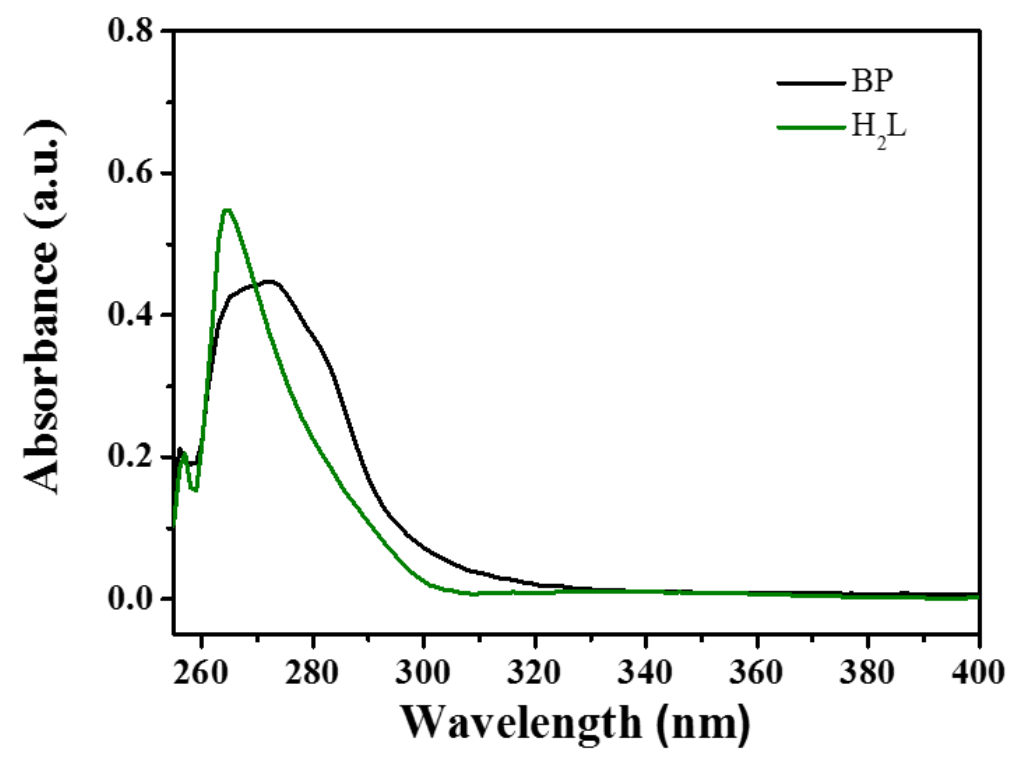

Figure S8 UV-vis absorption spectra of the $\mathrm{H}_{2} \mathrm{~L}$ ligand and BP.

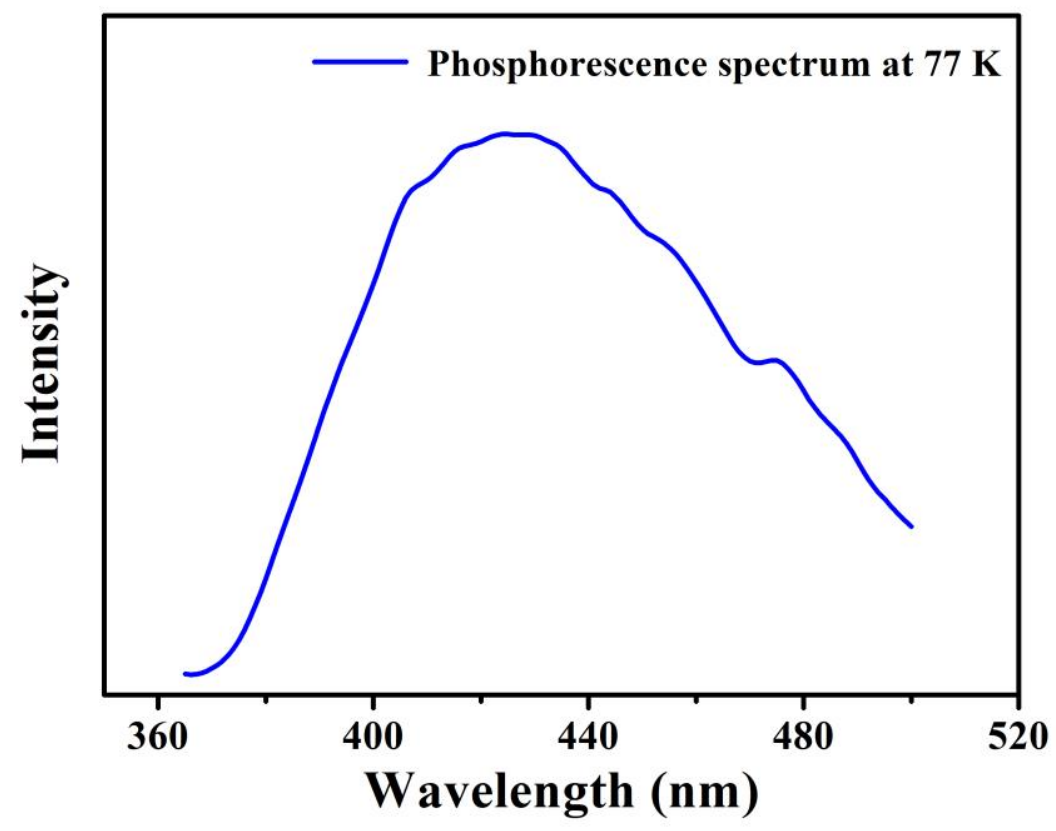

Figure S9 Phosphorescence spectrum of $\left\{\left[\mathrm{Gd}_{2} \mathrm{Zn}(\mathrm{L})_{4}\left(\mathrm{H}_{2} \mathrm{O}\right)_{8}\right] \cdot 8 \mathrm{H}_{2} \mathrm{O}\right\}_{\mathrm{n}}$ at $77 \mathrm{~K}$. 

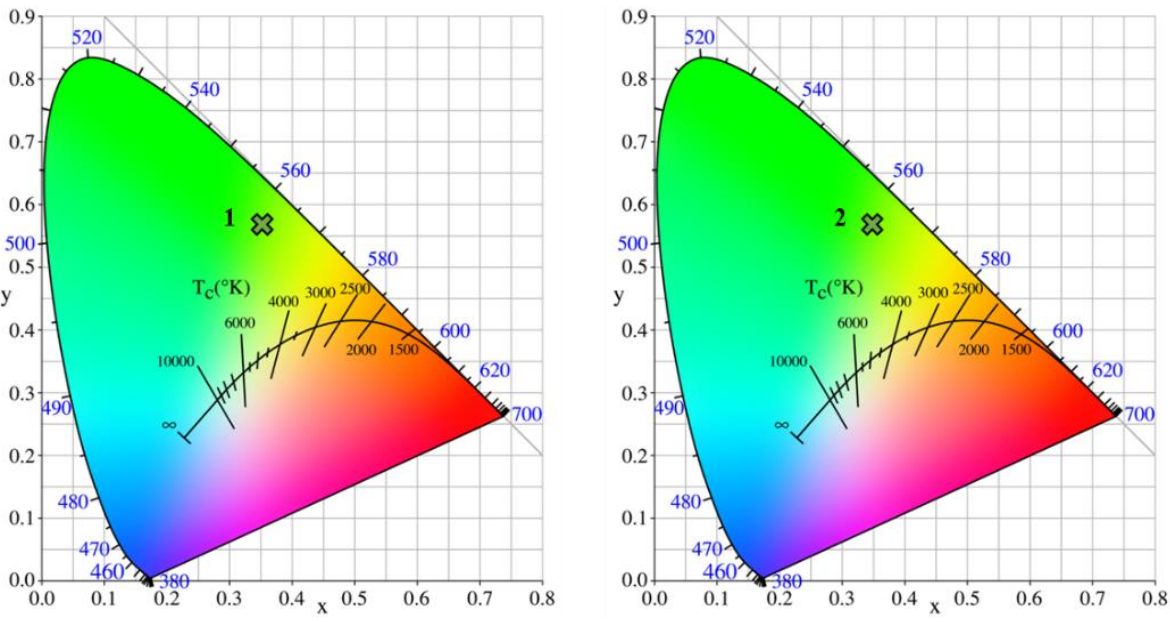

Figure S10 CIE 1931 chromaticity diagrams of 1 and 2.

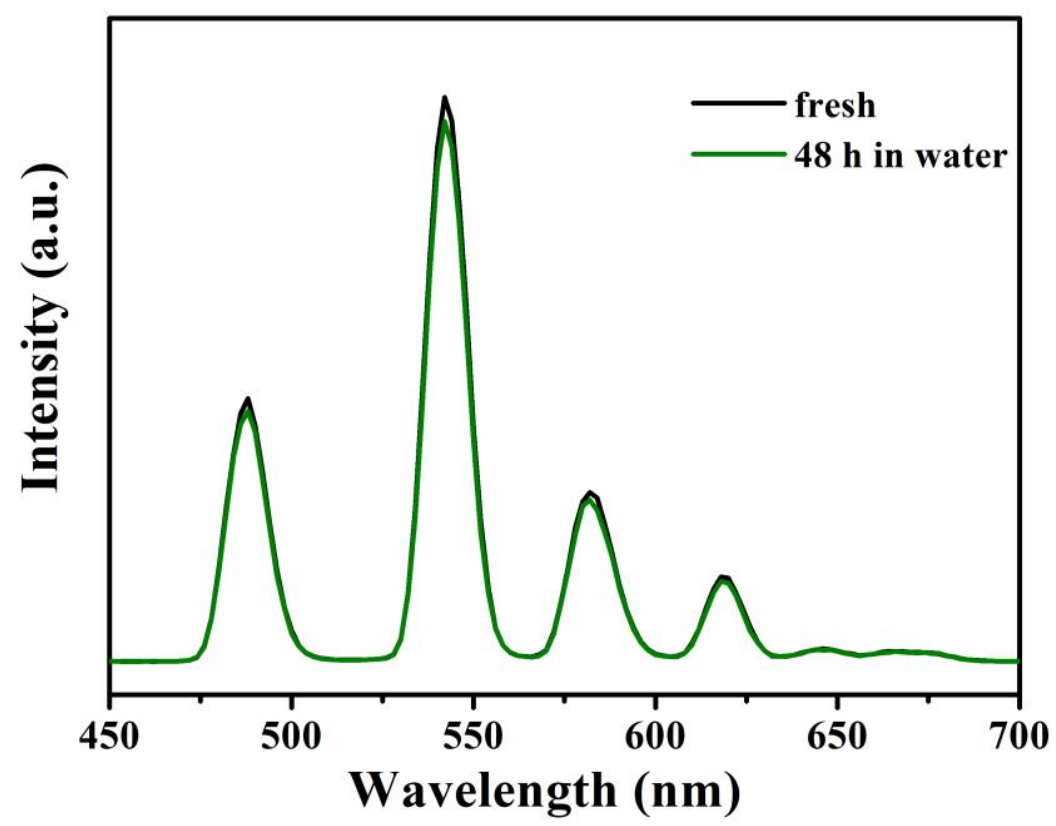

Figure S11 Emission spectra of $\mathbf{2}$ before and after storage in water for $48 \mathrm{~h}$. 
(a)

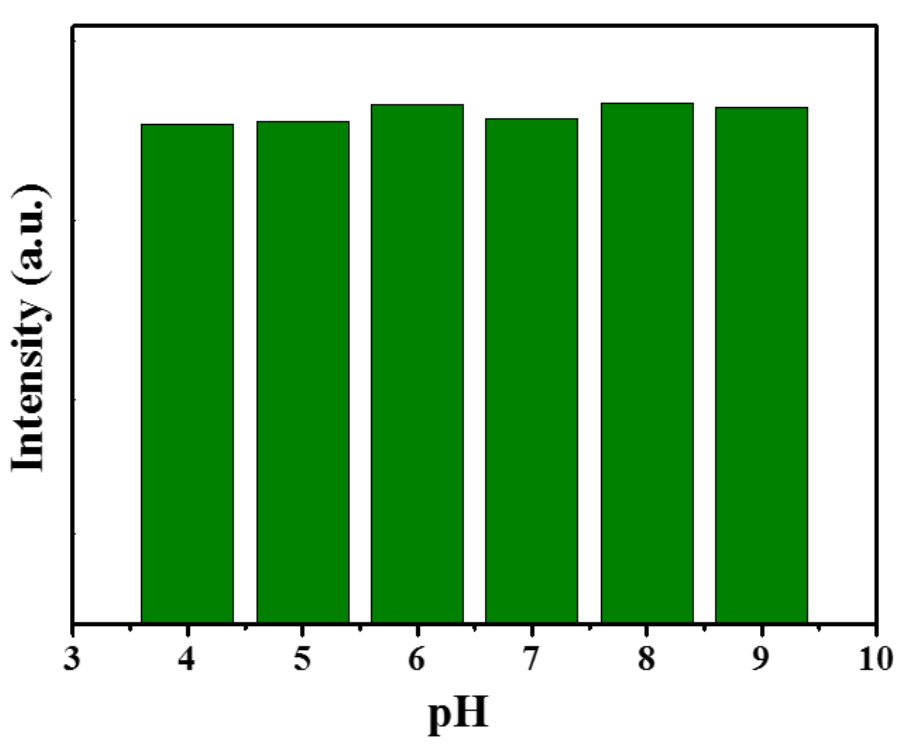

(b)

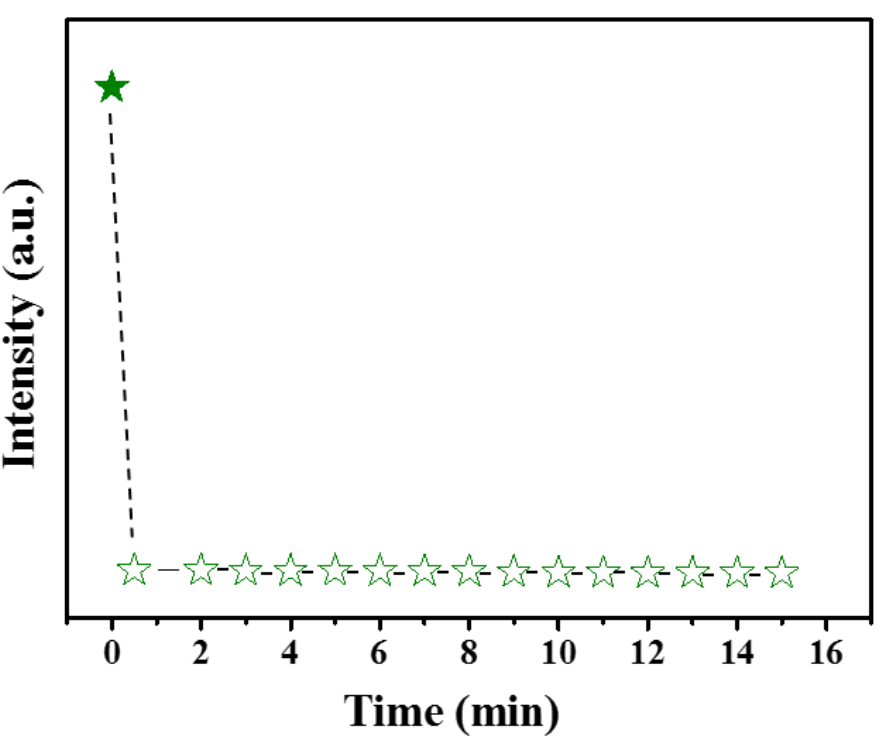

Figure S12 Emission intensities of 2 recorded at $\mathrm{pH}$ 4-9 with immersion in $\mathrm{HCl} / \mathrm{NaOH}$ solution (a) and various time intervals containing $\mathrm{BP}(\mathrm{b})$. 


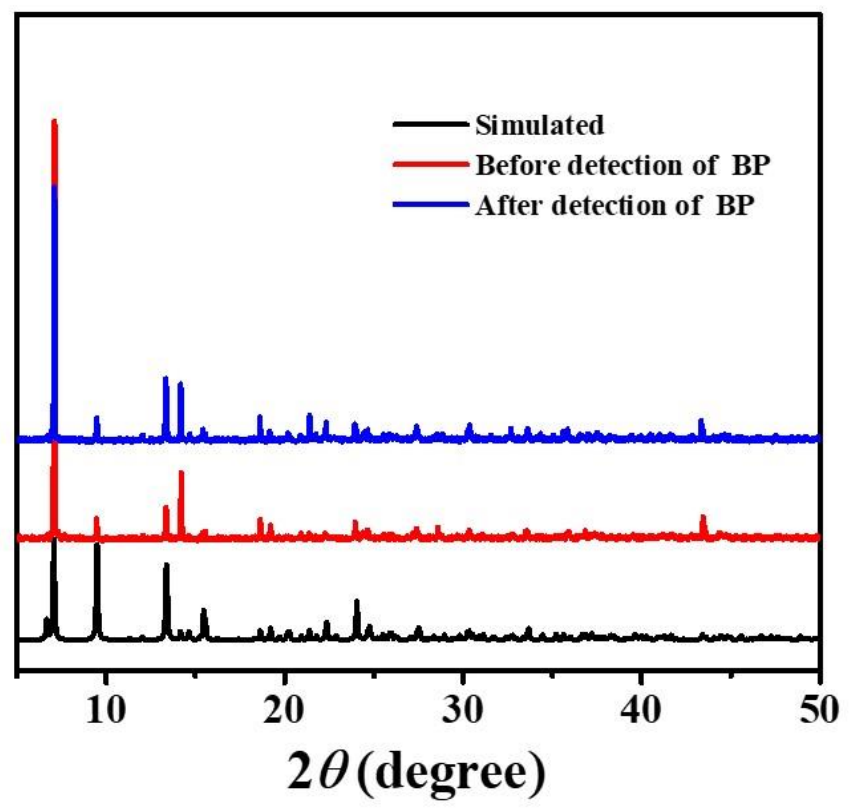

Figure S13 PXRD patterns of $\mathbf{2}$ before and after detection of BP. 
(a)

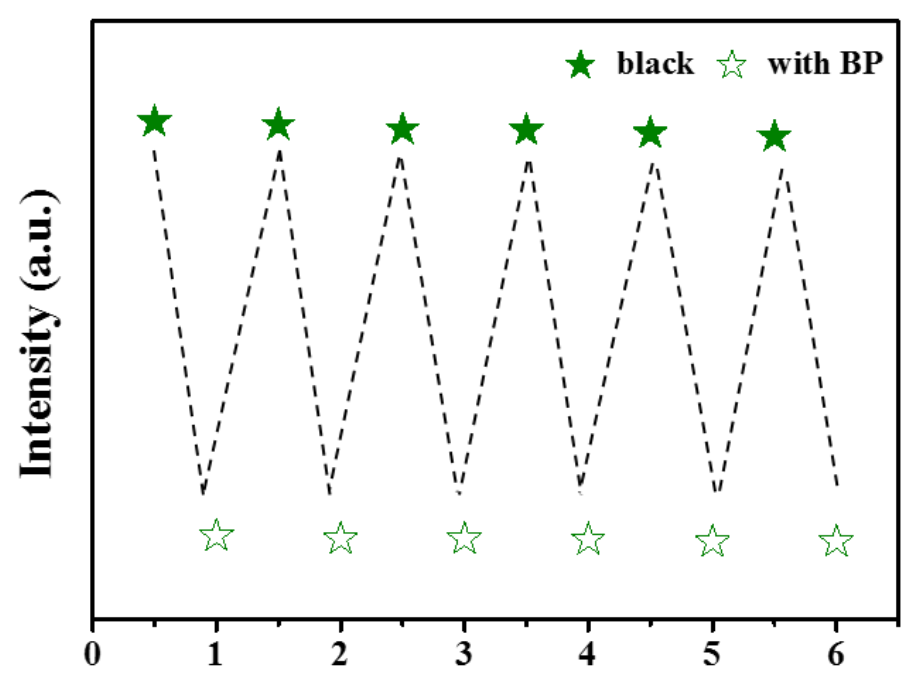

(b)

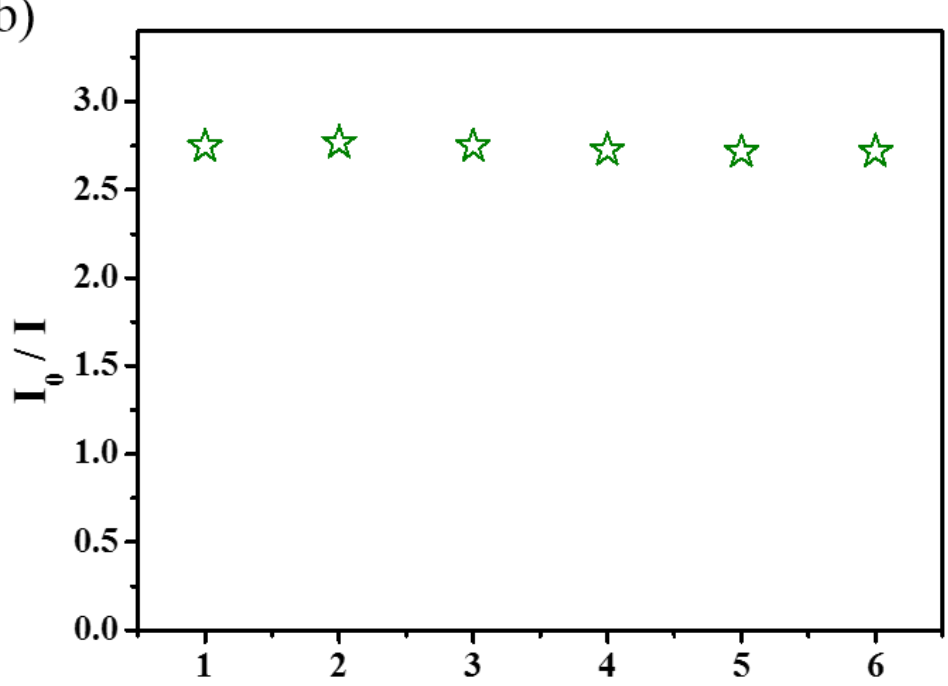

Figure S14 Reproducibility of the quenching ability of $\mathbf{2}$ dispersed in water in the presence of BP over six continuous quenching cycles at $546 \mathrm{~nm}$ (a), and the ratio of the luminescence intensity of $\mathbf{2}$ at $546 \mathrm{~nm}$ before and after addition of BP (b). 